\title{
Impulsive Control and Synchronization of Time-delay Chaotic System
}

\author{
Yan Yan ${ }^{1, a}$, Zhanji Gui ${ }^{2, b}$ and Kaihua Wang ${ }^{3, c}$ \\ ${ }^{1}$ Haikou College of Economics, Hainan,571127, China \\ ${ }^{2}$ Hainan College of Software Technology, Qionghai 571400, China \\ ${ }^{3}$ Hainan Normal University, Haikou, Hainan, 571158 ,China \\ a20689967@qq.com, ${ }^{b}$ zhanjigui@sohu.com, ${ }^{\text {c }}$ Corresponding Author: 35225454 @qq.com
}

Keywords: Impulsive system, Impulsive synchronization.

Abstract. With the increasingly perfecting and development of chaos theory, this paper considers the impulsive control and synchronization under the influence of time-delay chaotic system. It will utilize Lyapunov-krasovskii function and linear matrix inequality to research the asymptotic stability of chaotic deviation dynamical system between receiving system and sending system, and several new criteria will be obtained to ensure the impulsive control and synchronization of receiving and sending system.

\section{Introduction}

The research of chaos theory is a challenging and cross-subjects project with great potential applications and broad developing prospect. Especially since the 1990s, the breaking progress of chaos synchronization and control promote the research of chaos theory and also bring some opportunities and bright future of chaos application.

With the increasingly perfecting and development of chaos theory application, One new area comes out, that is the research of chaotic system under the Impulsive control and synchronization

(e.g[1]-[7]) .The Pulse design is established through Discrete time sequence driving system's state variable sample, and results the discrete of receiving system in the time sequence. After limited time, the path of two chaotic system will approach together,therefore these two chaotic system synchronise. However,in the Communication security system, the transmission lag in the application of the impulsive control and synchronization is unavoidable. It is necessary to analysis the robustness of Impulsive control and synchronization under the time-delay chaotic system. Therefore, this paper will consider the chaotic system with time-delay influences:

All manuscripts must be in English, also the table and figure texts, otherwise we cannot publish your paper.

$$
\dot{y}(t)=A y(t)+B y(t-\tau)+\sum_{i=1}^{n} f_{i}(y(t), t)+g(y(t-\tau), t)+u(t)
$$

Where $y(\cdot) \in R^{n}$ is state variable. $u(\cdot) \in R^{n}$ is control feedback in put variable, $A, B \in R^{n \times n}$ is constant matrix and $f_{i}, g \in R^{n}$ is nonlinear continuous function which ensure solution exists and is unique. $\tau>0$ is time-delay constant. This paper assume system (1) satisfies initial condition

$$
y(t)=\varphi(t) \quad t \in\left[t_{0}-\tau, t_{0}\right]
$$

Where $\varphi(t)$ in $\left[t_{0}-\tau, t_{0}\right]$ is bounded continuous

Assumesystem has unstable equilibrium or unstable periodic orbit $\tilde{y}(t)$, the purpose of feedback control is to ensure that $\tilde{y}(t)$ is asymptotic convergence.

This kind of control is a standard feedback control way,control input vector is:

$$
u(t)=K(\tilde{y}(t)-y(t))
$$

where $K$ at here is adjustable matrix coefficient of controller.

And then, it apply the pulse control system in Chaotic system (1): 


$$
\left\{\begin{array}{lr}
\dot{y}(t)=A y(t)+B y(t-\tau)+\sum_{i=1}^{n} f_{i}(y(t), t)+g(y(t-\tau), t)+u(t) \quad t \neq t_{i} \\
\Delta y(t)=y\left(t^{+}\right)-y\left(t^{-}\right)=D_{i} y\left(t^{-}\right) & t=t_{i}
\end{array}\right.
$$

where $y\left(t_{i}^{+}\right)=\lim _{t \rightarrow t_{i}^{+}} y(t), y\left(t_{i}^{-}\right)=\lim _{t \rightarrow t_{i}^{-}} y(t) \circ \mathrm{D}_{0}=0$.

Pulse time satisfy: $t_{0}<t_{1}<t_{2}<\cdots<t_{n}<t_{n+1}<\cdots$, and when $n \rightarrow \infty, t \rightarrow \infty$.

Assume $y\left(t_{i}^{-}\right)=y\left(t_{i}\right)(i=1,2, \ldots)$. The receiving end of driving system (1) is

$$
\tilde{y}(t)=\tilde{A y}(t)+B \tilde{y}(t-\tau)+\sum_{i=1}^{n} f_{i}(\tilde{y}(t), t)+g(\tilde{y}(t-\tau), t)
$$

$\tilde{y}(t)=\tilde{\varphi}(t), t \in\left[t_{0}-\tau, t_{0}\right]$ is initial conditionis. where $\tilde{\varphi}(t)$ in $\left[t_{0}-\tau, t_{0}\right]$ is bounded continuous.

In the discontinuity point $t_{i}(i=1,2, \ldots)$ the status which delivers from driving system will occur jump discontinuity. In this situation, the impulse model of driving system is:

$$
\begin{cases}\tilde{y}(t)=\tilde{A} \tilde{y}(t)+B \tilde{y}(t-\tau)+\sum_{i=1}^{n} f_{i}(\tilde{y}(t), t)+g(\tilde{y}(t-\tau), t) & t \neq t_{i} \\ \Delta \tilde{y}(t)=\tilde{y}\left(t^{+}\right)-\tilde{y}\left(t^{-}\right)=D_{i} \tilde{y}\left(t^{-}\right) & t=t_{i}\end{cases}
$$

Where $\tilde{y}\left(t_{i}^{+}\right)=\lim _{t \rightarrow t_{i}^{+}} \tilde{y}(t), \tilde{y}\left(t_{i}^{-}\right)=\lim _{t \rightarrow t_{i}^{-}} \tilde{y}(t) . \quad \mathrm{D}_{0}=0$, and $\tilde{y}(t)$ assume is leftcontinuous, then $y\left(t_{i}^{-}\right)=y\left(t_{i}\right)(i=1,2, \ldots)$.

According (3) and (5), it can obtain the error impulsive differential system is:

$$
\begin{cases}\dot{e}(t)=A e(t)+B e(t-\tau)+\sum_{i=1}^{n} F_{i}(e(t), t)+G(e(t-\tau), t)-K e(t) & t \neq t_{i} \\ \Delta e(t)=e\left(t^{+}\right)-e\left(t^{-}\right)=D_{i} e\left(t^{-}\right) & t=t_{i}\end{cases}
$$

Where

$$
e(t)=y(t)-\tilde{y}(t), F_{i}[e(t), t]=f_{i}[y(t), t]-f_{i}[\tilde{y}(t), t], G[e(t-\tau), t]=g[y(t-\tau), t]-[\tilde{y}(t-\tau), t] .
$$

The initial condition of error system (6) satisfy:

$$
e(t)=\varphi(t)-\tilde{\varphi}(t)=\phi(t) \quad t \in\left[t_{0}-\tau, t_{0}\right]
$$

since $\varphi(t), \tilde{\varphi}(t)$ is bounded continuous, therefore is bounded,

$$
\|\phi\|_{\tau}=\|\varphi-\tilde{\varphi}\|=\sup _{t \in\left[t_{0}-\tau, t_{0}\right]}\{\|\varphi(t)-\tilde{\varphi}(\mathrm{t})\|\}
$$

For the error system (6), due to the $F_{i}(0, t) \equiv 0, G(0, t) \equiv 0$, therefore it utilize Taylor expansion:

$$
\sum_{i=1}^{n} F_{i}(e(t), t)+G(e(t-\tau), t)=\sum_{i=1}^{n} A_{i}(t) e(t)+o(e(t))+A_{0}(t) e(t-\tau)+o(e(t-\tau))
$$

Here $A_{i}(t)=\left.\frac{\partial F_{i}[e(t), t]}{\partial e(t)}\right|_{e(t)=0}, A_{0}(t)=\left.\frac{\partial G[e(t-\tau), t]}{\partial e(t-\tau)}\right|_{e(t-\tau)=0}$.

Let

$$
o(e(t))+o(e(t-\tau))=H[e(t), e(t-\tau), t]=o(\|e(t)\|+\|e(t-\tau)\|)
$$

According to the infinitesimal of higher order conception, for all $\alpha_{i}>0(i=1,2)$, all exist $M>0$, when $\|e(t)\| \leq M$ and $\|e(t-\tau)\| \leq M$, then:

$$
H[e(t), e(t-\tau), t] \leq \alpha_{1}\|e(t)\|+\alpha_{2}\|e(t-\tau)\|
$$

Assume $A_{i}(t)=A_{i 1}+A_{i 2}(t)(t=0,1,2, \ldots$.$) , where A_{i 1}$ is constant matrix, $A_{i 2}(t)$ is time-varying uncertain matrix, which satisfy:

$$
\left\|A_{i 2}(t)\right\| \leq \beta_{i}
$$

where $\beta_{i}(i=0,1,2, \ldots)$ is constant. 
According to the above assumption, system(6) can rewrite as:

$$
\begin{cases}\dot{e}(t)=E e(t)+F e(t-\tau)+\sum_{i=1}^{n} A_{i 2}(t) e(t)+A_{02}(t) e(t-\tau) & \\ \quad+H[e(t), e(t-\tau), t] & t \neq t_{i} \\ \Delta e(t)=e\left(t^{+}\right)-e\left(t^{-}\right)=D_{i} e\left(t_{i}^{-}\right) & t=t_{i} \\ e(t)=\phi(t) & t \in\left[t_{0}-\tau, t_{0}\right]\end{cases}
$$

where $E=A-k+\sum_{i=1}^{n} A_{i 1}, F=B+A_{01}, D_{0}=0$.

Due to $\left[\mathrm{F} \int_{t-\tau}^{t} e(s) d s\right]^{\prime}=F e(t)-F e(t-\tau)$, then system (12) can be changed as:

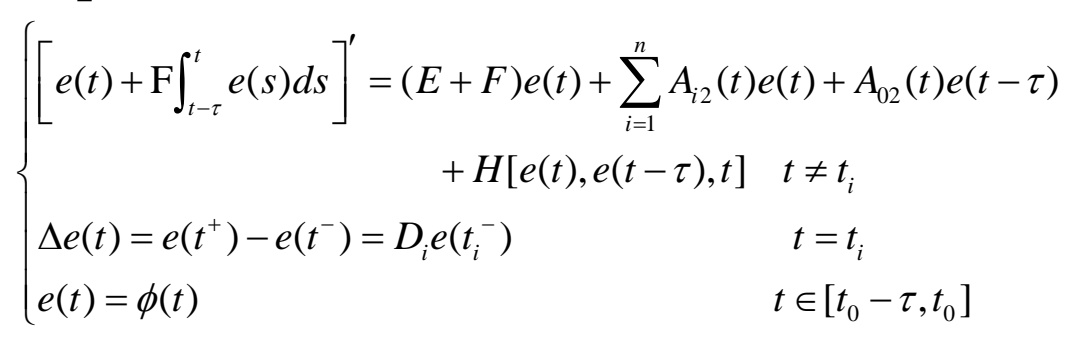

For the sake of convenient, it define $L: C\left([-\tau, 0], R^{n}\right) \rightarrow R^{n}$ as:

$$
L\left(e_{t}\right)=e(t)+F \int_{t-\tau}^{t} e(s) d s
$$

Before this paper put forward the main conclusion, there introduce three lemmas:

Lemmas 1: For the vector $a, b \in R^{n}$ and symmetric positivematrix $Q$, there is:

$$
2 a^{T} b \leq a^{T} Q^{-1} a+b^{T} Q b
$$

Lemmas 2: For any positive matrix $N \in R^{n \times n}$, it exists $\gamma>0$, and vector function $\omega:[0, \gamma] \rightarrow R^{n}$, there is: $\quad\left(\int_{0}^{y} \omega(s) d s\right)^{T} N\left(\int_{0}^{y} \omega(s) d s\right) \leq \gamma \int_{0}^{y} \omega^{T}(s) N \omega(s) d s$

Lemmas 3: ( Schur Complement) exists constant matrix $Z_{1}, Z_{2}, Z_{3}$, where $Z_{1}=Z_{1}^{T}, Z_{2}=Z_{2}^{T}$, there is: $\left(\begin{array}{cc}Z_{1} & Z_{3}^{T} \\ Z_{3} & Z_{2}\end{array}\right)<0$, if and only if $Z_{1}<0, Z_{2}-Z_{3} Z_{1}^{-1} Z_{3}^{T}<0$; or $Z_{2}<0, Z_{2}-Z_{3} Z_{2}^{-1} Z_{3}^{T}<0$.

\section{Impulsive control and synchronization with time-delay and chaotic system}

Theorem1: For system (13), if it satisfy $\tau\|F\|<1$, and exist positivesymmetric matrix $P>0, Q>0$, which make the linear matrix inequality satisfy:

$$
\begin{aligned}
& N=\left(\begin{array}{cc}
(E+F)^{T} P+P(E+F)+L_{1} I+L_{2} I+L_{3} I+L_{4} I+4 Q \tau & \tau(E+F)^{T} P F \\
\tau F^{T} P(E+F) & -\tau Q
\end{array}\right)<0 \\
& \left\|1+D_{i}\right\| \sqrt{\frac{(1+\tau\|F\|)^{2} \lambda_{\text {max }}(P)+\tau\left(L_{1}+L_{2}\right)+2 \tau^{2} \lambda_{\text {max }} Q}{(1-\tau\|F\|)^{2} \lambda_{\text {min }}(P)}}<1
\end{aligned}
$$

Then the zero-solution of error sytem(13) is exponentially stable, that is mean the pulsedindex of system (1) and system (4) is synchronous, where $L_{1}, L_{2}, L_{3}, L_{4}$, can be viewed respectivelyas:

$$
L_{1}=\tau \beta_{0}^{2}\left\|P F Q^{-1} F P\right\|+\beta_{0}\|P\|, \quad L_{2}=\left(\alpha_{1}+\alpha_{2}\right) \alpha_{2}\left\|P F Q^{-1} F^{T} P\right\|+\alpha_{2}\|P\|
$$




$$
\begin{aligned}
& L_{3}=2 \sum_{i=1}^{n} \beta_{i}\|P\|+\tau \sum_{i=1}^{n} \beta_{i}^{2}\left\|P F Q^{-1} F^{T} P\right\|+\beta_{0}\|P\| \\
& L_{4}=\left(\alpha_{1}+\alpha_{2}\right) \alpha_{1}\left\|P F Q^{-1} F^{T} P\right\|+\alpha_{2}\|P\|+2 \alpha_{1}\|P\|
\end{aligned}
$$

\section{Proof:}

According to the definition's condition (15), for $\varepsilon>0$, let:

$$
N+\left(e^{\varepsilon \tau}-1\right)\left(\begin{array}{cc}
L_{1} I+L_{2} I+4 Q \tau & 0 \\
0 & 0
\end{array}\right)+\varepsilon e^{\varepsilon \tau}\left(\begin{array}{cc}
\tau Q+\tau^{2} F^{T} P F & 0 \\
0 & 0
\end{array}\right)+\varepsilon\left(\begin{array}{cc}
P & P F \\
F^{T} P & Q^{-1}
\end{array}\right) \leq 0
$$

Let Lyapunov-Krasovskii function as :

$$
\begin{aligned}
& V\left(e_{t}\right)=V_{1}\left(e_{t}\right)+V_{2}\left(e_{t}\right)+V_{3}\left(e_{t}\right), \quad V_{1}\left(e_{t}\right)=L^{T}\left(e_{t}\right) P L\left(e_{t}\right) e^{\varepsilon t}, \\
& V_{2}\left(e_{t}\right)=\int_{-\tau}^{0} \int_{t+s}^{t} e^{T}(\rho)\left[(4+\varepsilon) Q+\varepsilon \tau F^{T} P F\right] e(\rho) e^{\varepsilon(\rho+\tau)} d \rho d s, V_{3}\left(e_{t}\right)=\left(L_{1}+L_{2}\right) \int_{t-\tau}^{t}\|e(s)\|^{2} e^{\varepsilon(s+\tau)} d s .
\end{aligned}
$$

Through system (13), to make a derivative ofLyapunov-Krasovskii function $V_{1}\left(e_{t}\right)$, obtain:

$$
\begin{aligned}
\frac{d V_{1}\left(e_{t}\right)}{d t}= & 2 e^{\varepsilon t} L^{T}\left(e_{t}\right) P \frac{d L\left(e_{t}\right)}{d t}+\varepsilon e^{\varepsilon t} L^{T}\left(e_{t}\right) P L\left(e_{t}\right) \\
= & e^{\varepsilon t}\left\{e^{T}(t)\left[P\left(F+E+\sum_{i=1}^{n} A_{i 2}(t)\right)\right]+\left(F+E+\sum_{i=1}^{n} A_{i 2}(t)\right)^{T} P\right] e(t) \\
& +2 e^{T}(t) P A_{02}(t) e(t-\tau)+2 e^{T}(t)(E+F)^{T} P F \int_{t-\tau}^{t} e(s) d s \\
& +2 e^{T}(t-\tau) A_{02}{ }^{T}(t) P F \int_{t-\tau}^{t} e(s) d s+2 e^{T}(t)\left(\sum_{i=1}^{n} A_{i 2}(t)\right)^{T} P F \int_{t-\tau}^{t} e(s) d s \\
& +2 e^{T}(t) P H[e(t), e(t-\tau), t]+2 H^{T}[e(t), e(t-\tau), t] P F \int_{t-\tau}^{t} e(s) d s \\
& \left.+\varepsilon\left[e^{T}(t) P e(t)+2 e^{T}(t) P F \int_{t-\tau}^{t} e(s) d s+\int_{t-\tau}^{t} e^{T}(s) d s F^{T} P F \int_{t-\tau}^{t} e(s) d s\right]\right\}
\end{aligned}
$$

To apply Lemmas 1 and system (11), it can be obtained:

$$
\begin{aligned}
\frac{d V_{1}\left(e_{t}\right)}{d t} \leq & e^{\varepsilon t}\left\{e^{T}(t)\left[P\left(F+E+\sum_{i=1}^{n} \beta_{i} I\right)\right]+\left(F+E+\sum_{i=1}^{n} \beta_{i} I\right)^{T} P\right] e(t) \\
& +2 e^{T}(t) P A_{02}(t) e(t-\tau)+2 e^{T}(t)(E+F)^{T} P F Q^{-1} F^{T} P(E+F) e(t) \\
& +\tau \beta_{0}^{2} e^{T}(t-\tau) P F Q^{-1} F^{T} P e(t-\tau)+4 \int_{t-\tau}^{t} e^{T}(s) Q e(s) d s+\tau \sum_{i=1}^{n} \beta_{i}^{2} e^{T}(t) P F Q^{-1} F^{T} P e(t) \\
& +2 e^{T}(t) P H[e(t), e(t-\tau), t]+2 H^{T}[e(t), e(t-\tau), t] P F Q^{-1} F^{T} P H[e(t), e(t-\tau), t] \\
& \left.+\varepsilon\left[e^{T}(t) P e(t)+2 e^{T}(t) P F \int_{t-\tau}^{t} e(s) d s+\int_{t-\tau}^{t} e^{T}(s) d s F^{T} P F \int_{t-\tau}^{t} e(s) d s\right]\right\}
\end{aligned}
$$

When $\|\phi\|<M$, there exist $l>0$, which make $\forall t \in\left[t_{0}, t_{0}+l\right)$ has $\|e(t)\| \leq M$, $\|e(t-\tau)\| \leq M$, now satisfy system(10), and then apply Lemma 2, here it is:

$$
\begin{aligned}
\frac{d V_{1}\left(e_{t}\right)}{d t} \leq & e^{\varepsilon t}\left\{e^{T}(t)\left[P\left(F+E+\sum_{i=1}^{n} \beta_{i} I\right)\right]+\left(F+E+\sum_{i=1}^{n} \beta_{i} I\right)^{T} P\right] e(t) \\
& +4 \int_{t-\tau}^{t} e^{T}(s) Q e(s) d s+\tau e^{T}(t)(E+F)^{T} P F Q^{-1} F^{T} P(E+F) e(t) \\
& +\tau \sum_{i=1}^{n} \beta_{i}^{2}\left\|P F Q^{-1} F^{T} P\right\|\|e(t)\|^{2}+\tau \beta_{0}^{2}\left\|P F Q^{-1} F^{T} P\right\|\|e(t-\tau)\|^{2} \\
& +2 \beta_{0}\|P\|\|e(t)\|\|e(t-\tau)\|+2 \alpha_{1}\|P\|\|e(t)\|^{2}
\end{aligned}
$$




$$
\begin{aligned}
& +2 \alpha_{2}\|P\|\|e(t)\|\|e(t-\tau)\|+\left\|P F Q^{-1} F^{T} P H\right\|\left(\alpha_{1}\|e(t)\|+\alpha_{2}\|e(t-\tau)\|\right)^{2} \\
& \left.+\varepsilon\left[e^{T}(t) P e(t)+e^{T}(t) P F Q^{-1} F^{T} P e(t)+\int_{t-\tau}^{t} e^{T}(s) Q e(s) d s+\int_{t-\tau}^{t} e^{T}(s) F^{T} P F e(s) d s\right]\right\}
\end{aligned}
$$

Therefore, to make a derivative of function $V_{1}\left(e_{t}\right)$ with system (13) , it obtain:

$$
\begin{aligned}
\frac{d V_{1}\left(e_{t}\right)}{d t} \leq & e^{\varepsilon t}\left\{e ^ { T } ( t ) \left[P(F+E)+(F+E)^{T} P+(E+F)^{T} P F Q^{-1} F^{T} P(E+F)\right.\right. \\
& \left.+\varepsilon P+\varepsilon P F Q^{-1} F^{T} \mathrm{P}+L_{3} I+L_{4} I\right] e(t)+\varepsilon \int_{t-\tau}^{t} e^{T}(s) F^{T} P e(s) d s \\
& \left.+(4+\varepsilon) \int_{t-\tau}^{t} e^{T}(s) Q e(s) d s+\left(L_{1}+L_{2}\right)\|e(t-\tau)\|\right\}
\end{aligned}
$$

For

$$
\begin{gathered}
\frac{d V_{2}\left(e_{t}\right)}{d t} \leq e^{\varepsilon t} \tau e^{T}(t)\left[(4+\varepsilon) Q+\varepsilon \tau F^{T} P F\right] e(t)-e^{\varepsilon t} \int_{t-\tau}^{t} e^{T}(s)\left[(4+\varepsilon) Q+\varepsilon \tau F^{T} P F\right] e(s) d s \\
\frac{d V_{3}\left(e_{t}\right)}{d t}=\left(L_{1}+L_{2}\right)\|e(t)\|^{2} e^{\varepsilon(t+\tau)}-\left(L_{1}+L_{2}\right)\|e(t-\tau)\|^{2} e^{\varepsilon t}
\end{gathered}
$$

Accord to system (18), (19), (20), there is :

$$
\begin{aligned}
\frac{d V\left(e_{t}\right)}{d t} & \leq e^{\varepsilon \tau}\left\{e^{T}(t)\left(P(E+F)+(E+F)^{T} P+\tau(E+F)^{T} P F Q^{-1} F^{T} P(E+F)\right)\right. \\
& \left.\left.+\left(L_{1} I+L_{2} I\right) e^{\varepsilon \tau}+L_{3} I+L_{4} I+\tau(4+\varepsilon) e^{\varepsilon \tau} Q+\varepsilon \tau^{2} e^{\varepsilon \tau} F^{T} P F+\varepsilon P+P F Q^{-1} F^{T} P\right) e(t)\right\}
\end{aligned}
$$

For $\forall t \in\left[t_{0}, t_{0}+l\right)$, according to the inequation (17), there is :

$$
V^{\prime}\left(e_{t}\right) \leq 0
$$

for $\forall t \in\left[t_{0}, t_{1}\right]$, there is :

$$
\begin{gathered}
V\left(e_{t}\right) \leq V\left(e_{t_{0}}\right)=\leq \lambda_{\max }(P)(1-\tau\|F\|)^{2}\|\phi\|_{\tau}^{2} e^{\varepsilon t_{0}}+\tau\left(L_{1}+L_{2}\right) e^{\varepsilon \tau} e^{\varepsilon t_{0}}\|\phi\|_{\tau}^{2} \\
+\lambda_{\max }\left[(4+\varepsilon) Q+\varepsilon \tau F^{T} P F\right] \frac{\tau^{2}}{2} e^{\varepsilon \tau} e^{\varepsilon t_{0}}\|\phi\|_{\tau}^{2}=C e^{\varepsilon t_{0}}\|\phi\|_{\tau}^{2}
\end{gathered}
$$

where

$$
C=\lambda_{\max }(P)(1-\tau\|F\|)^{2}\|\phi\|_{\tau}^{2} e^{\varepsilon t_{0}}+\tau\left(L_{1}+L_{2}\right) e^{\varepsilon \tau}+\lambda_{\max }\left[(4+\varepsilon) Q+\varepsilon \tau F^{T} P F\right] \frac{\tau^{2}}{2} e^{\varepsilon \tau} .
$$

Frome literature (8), it is:

$$
(1-\tau\|F\|)^{2}\|e(t)\|^{2} \leq\left\|L\left(e_{t}\right)\right\|^{2},
$$

and then

$$
(1-\tau\|F\|)^{2} \lambda_{\text {min }}(P)\|e(t)\|^{2} e^{\varepsilon t} \leq L^{T}\left(e_{t}\right) P L\left(e_{t}\right) e^{\varepsilon t} \leq V\left(e_{t}\right) \leq C\|\phi\|^{2} e^{\varepsilon t_{0}} .
$$

That is

$$
\|e(t)\| \leq \sqrt{\frac{C}{(1-\tau\|F\|)^{2} \lambda_{\text {min }}(P)}}\|\phi\|_{\tau} e^{-\frac{\varepsilon}{2}\left(t-t_{0}\right)}=\Phi\|\phi\|_{\tau} e^{-\frac{\varepsilon}{2}\left(t-t_{0}\right)} \quad t \in\left[t_{0}, t_{1}\right),
$$

where

$$
\Phi=\sqrt{\frac{C}{(1-\tau\|F\|)^{2} \lambda_{\text {min }}(P)}},
$$

according to the system (13),it can deduct that:

$$
\left\|e\left(t_{1}^{+}\right)\right\|=\left\|\left(1+D_{1}\right) e\left(t_{1}\right)\right\| \leq\left\|\left(1+D_{1}\right)\right\| \Phi\|\phi\|_{\tau} e^{-\frac{\varepsilon}{2}\left(t-t_{0}\right)},
$$

according to system (21), $\forall t \in\left[t_{0}, t_{1}\right]$, there is

$$
V\left(e_{t}\right) \leq V\left(e_{t_{1}^{+}}\right) \leq C\left\|e\left(t_{1}^{+}\right)\right\|^{2} e^{\varepsilon t_{1}} \leq C\left\|\left(1+D_{1}\right)\right\|^{2} \Phi\|\phi\|_{\tau}^{2} e^{\varepsilon \tau} e^{-\varepsilon\left(t_{1}-t_{0}\right)} e^{\varepsilon t_{1}},
$$

for 


$$
(1-\tau\|F\|)^{2} \lambda_{\min }(P)\|e(t)\|^{2} e^{\varepsilon t} \leq L^{T}\left(e_{t}\right) P L\left(e_{t}\right) e^{\varepsilon t} \leq V\left(e_{t}\right) \leq C\left\|\left(1+D_{1}\right)\right\|^{2} \Phi\|\phi\|_{\tau}^{2} e^{\varepsilon \tau} e^{-\varepsilon\left(t_{1}-t_{0}\right)} e^{\varepsilon t_{1}},
$$
so $\|e(t)\| \leq\left\|\left(1+D_{1}\right)\right\| \Phi^{2}\|\phi\|_{\tau} e^{\frac{\varepsilon \tau}{2}} e^{-\frac{\varepsilon}{2}\left(t-t_{0}\right)} \quad t \in\left(t_{1}, t_{2}\right]$.

by utilizing mathematicalinduction $\forall t \in\left(t_{k}, t_{k+1}\right]$, there is:

$$
\left\|e\left(t_{k+1}^{+}\right)\right\| \leq \prod_{i=1}^{k+1}\left\|\left(1+D_{i}\right)\right\| \Phi^{k+1}\|\phi\|_{\tau} e^{\frac{k \varepsilon \tau}{2}} e^{-\frac{\varepsilon}{2}\left(t_{k+1}-t_{0}\right)},
$$

when $\varepsilon>0$, according to the condition(16) can be known:

$$
\left\|1+D_{i}\right\| \sqrt{\frac{C}{(1-\tau\|F\|)^{2} \lambda_{\min }(P)}} e^{\frac{\varepsilon \tau}{2}}=\left\|1+D_{i}\right\| \Phi e^{\frac{\varepsilon \tau}{2}} \leq 1,
$$

if $d=\max \{1, \Phi\}, \forall t \geq t_{0}$ all have:

$$
\|e(t)\| \leq d\|\phi\| e^{-\frac{\varepsilon}{2}\left(t-t_{0}\right)}
$$

When $\|\phi\| \leq \frac{M}{d}$, it is easy to get $\forall t \in\left[t_{0}, \infty\right)$, and all $\|e(t)\| \leq M,\|e(t-\tau)\| \leq M$, therefore, for $\forall t \in\left[t_{0}, \infty\right)$, ineqution (22) is established. therefore, the zero solution of error system (13) is exponentially stable, thus thepulsed index of system (1) and system (4) is synchronous.

\section{Acknowledgements}

This work is supported jointly by the Natural Sciences Foundation of China under Grant No. 60963025, Natural Sciences Foundation of Hainan Province under Grant No. 110007, Haikou College of Economics No.hjky16-17 and the Start-up fund of Hainan Normal University under Project No. 00203020201.

\section{References}

[1] Moosa Ayati, Hamid Khaloozadeh, Xinzhi Liu, "Synchronizing impulsive synchronization chaotic systems with parametric uncertainty via a novel adaptive impulsive observer", Asian Journal of Control, vol.13, no. 6, pp. 809-817, 2011

[2] Jin Huan, Li Chuandong, "Chaotification of Linear Dynamical System via Impulsive Control", IJIPM: International Journal of Information Processing and Management, Vol. 2, No. 3, pp. 27 33, 2011

[3] Ming Zhao, XueFei Wu, Chen Xu, "Exponential Synchronization of Delayed Complex Networks with Stochastic Perturbations via Impulsive Control", JCIT, Vol. 7, No. 16, pp. 115 -125, 2012

[4] A. Khadra, X. Z. Liu, X. Shen, "Robust impulsive synchronization and its application to communication security”, Int. J. Dyn. Continuous Discr. Impulsive Syst, vol.10, no, 3, pp.403-416, 2003

[5] Liu Z, Liao L, "Existence and global exponential stability of periodic solution of cellular neural networks with time-varying delays”, J. Math. Anal. Appl. , vol.290, no. 1, pp. 247-262, 2004

[6] Amritkar R E,Gupte N.Synchronization of chaotic orbits: the effect of a finite time steps Physical Review E,47(6), 3889-3895, 1993

[7] Suykens J A., Yang T, Chua L O.impulsive synchronization of chaotic Lur' e systems by measurement feedback. International Journal of Bifurcation and Chaos,8(6):1371-1381, 1998

[8] V.Kolmanovskii, A.Myshkis.introduction to the Theory and application of Functional differential Equations.Luwer Academic Publishers, 527 